\title{
THE ROLE OF PROPHYLACTIC ANTIBIOTICS IN PATIENTS UNDERGOING ELECTIVE CHOLECYSTECTOMY- A RANDOMISED PLACEBO CONTROLLED RECIPIENT BLIND TRIAL
}

\author{
Shibaji Basu¹, Pankaj Kumar Sarkar², Shibsankar Ray Chaudhury33, Souryav Sankar Mondal 4 \\ ${ }^{1}$ RMO-Cum-Clinical Tutor, Department of General Surgery, Medical College Hospital, Kolkata, West Bengal. \\ ${ }^{2}$ Assistant Professor, Department of General Surgery, Medical College Hospital, Kolkata, West Bengal. \\ ${ }^{3}$ Associate Professor, Department of General Surgery, Medical College Hospital, Kolkata, West Bengal. \\ ${ }^{4}$ Postgraduate Trainee, Department of General Surgery, Medical College Hospital, Kolkata, West Bengal.
}

\begin{abstract}
\section{BACKGROUND}

Laparoscopic cholecystectomy (LC) is the gold standard treatment for symptomatic cholelithiasis. The incidence of infectious complications after LC is significantly lower compared with infections with open cholecystectomy.1,2 Antibiotic prophylaxis may not be necessary in low-risk patients undergoing elective LC. ${ }^{6-10}$ On the contrary, many other surgeons still use and recommend the administration of prophylactic antibiotics. ${ }^{11-15}$

The aim of this study is to determine the necessity of prophylactic antibiotics in preventing the postoperative infective complications in patients undergoing elective laparoscopic cholecystectomy.
\end{abstract}

\section{MATERIALS AND METHODS}

Patients who have abdominal symptoms with cholelithiasis, confirmed by ultrasonography, willing for operation and giving consent for the study formed the study population. The study included 80 patients undergoing laparoscopic cholecystectomy. The patients were randomised according to a computer generated block randomisation schedule, to receive either (1) Three doses of Cefazolin (Group A; $n=40$ ), one just after the induction of anaesthesia and two after the operation or (2) Physiologic saline as placebo (Group B; $n=40$ ) after the induction of anaesthesia. Patient demographics and clinical and surgical outcomes (infective complications mainly) will be recorded.

\section{RESULTS}

Both groups were comparable with reference to age and sex distribution ( $p$ values were not significant). 6 patients out the total patients developed superficial wound infection, which were managed by suture removal at the site of purulent discharge and regular dressing at home. 2 out of the 6 patients belonged to group A, while 4 belonged to group B. There was no significant difference in wound infection rates in both the groups. There was no association between intraoperative biliary spillage and superficial wound infection. Umbilicus was the site of wound infection in all 6 patients.

\section{CONCLUSION}

Antibiotic prophylaxis is not necessary in low-risk patients with gallstone disease undergoing elective laparoscopic cholecystectomy to prevent postoperative infection-related complications.

\section{KEYWORDS}

Laparoscopic Cholecystectomy, Surgical Site Infection, Prophylactic Antibiotics.

HOW TO CITE THIS ARTICLE: Basu S, Sarkar PK, Chaudhury SR, et al. The role of prophylactic antibiotics in patients undergoing elective cholecystectomy- A randomised placebo controlled recipient blind trial. J. Evolution Med. Dent. Sci. 2017;6(48):3676-3680, DOI: $10.14260 /$ Jemds $/ 2017 / 794$

\section{BACKGROUND}

Laparoscopic cholecystectomy (LC) is the gold standard treatment for symptomatic cholelithiasis. The incidence of infectious complications after LC is significantly lower compared with infections with open cholecystectomy.1,2 The infective complications of open cholecystectomy are well known, and prophylactic antibiotics are a routine practice. However, the wounds created after open cholecystectomy behave differently as compared to laparoscopic cholecystectomy.

Financial or Other, Competing Interest: None.

Submission 03-04-2017, Peer Review 03-06-2017,

Acceptance 08-06-2017, Published 15-06-2017.

Corresponding Author:

Dr. Pankaj Kumar Sarkar,

\#334/4, Dr. M. N. Saha Road,

Dum Dum, Kolkata-700074,

West Bengal.

E-mail: pankajkrsarkar15@gmail.com

DOI: $10.14260 /$ jemds $/ 2017 / 794$

First, the wounds created are smaller in LC as compared to the open surgery. Secondly, it has been proved that the immune system is better preserved in laparoscopic surgery since the tissue trauma is less. ${ }^{3}$ These result in lesser activation of the inflammatory response following the laparoscopic procedure. ${ }^{3}$ Furthermore, laparoscopic cholecystectomy per se does not violate the mucosal defence barrier of the respiratory, gastrointestinal or genital epithelium. Observing the low incidence of infections following laparoscopic cholecystectomy, the need for antibiotics is now frequently questioned.

The benefits of preoperative antibiotics, which reduce bacterial contamination during clean-contaminated operations such as cholecystectomy and contaminated operations, are well known.4-6 Preventing postoperative infection is an essential factor in improving the results of surgical procedures. One approach to preventing infection is the administration of prophylactic antibiotics. The use of prophylactic antibiotics as a means of preventing surgical site 
infections (SSIs) is still controversial in elective LC, which has a low risk for infectious complications.

Antibiotic prophylaxis may not be necessary in low-risk patients undergoing elective LC. ${ }^{7-11}$ Various factors, including the small incisions, minimal tissue handling, low impact on the immune system, little exposure to the external environment, $\mathrm{CO} 2$ pneumoperitoneum, and better visibility of tissues for dissection and haemostasis, have been cited. The over-use of antibiotics can result in a rising frequency of adverse effects, emergence of drug-resistant organisms, as well as increased cost.12-13 On the contrary, many other surgeons still use and recommend the administration of prophylactic antibiotics. ${ }^{14-18}$

Because of its broad-spectrum antimicrobial effect, low toxicity, and low cost, the single-dose use of cefazolin has been recommended for patients undergoing open cholecystectomy and other biliary surgeries. ${ }^{5}$ As controversy still surrounds the routine use of prophylactic antibiotics in elective LC, this study is being conducted with the aim of investigating the necessity and testing the efficacy of Cefazolin (triple dose) as a prophylactic antibiotic to prevent postoperative infective complications in low-risk patients undergoing LC.

\section{MATERIALS AND METHODS}

A single blind, prospective, randomised, placebo controlled study comparing the prophylactic use of cefazolin (Group A) vs. placebo (Group B) was performed between January 1, 2015 and June 31, 2016 in Department of Surgery, Medical College and Hospital, Kolkata. 80 patients undergoing elective laparoscopic cholecystectomy were selected as suitable for the study protocol. All patients participating in the study gave informed written consent. Patients who have abdominal symptoms/incidentally detected patients with cholelithiasis, confirmed by ultrasonography, willing for operation and giving consent for the study formed the inclusion criteria.

\section{Exclusion Criteria}

1. Patients not willing to giving consent for the study.

2. Peptic ulcer, any other causes of pain like perforation or obstruction in the abdomen, acute cholecystitis, evidence of obstructive jaundice and/or cholangitis.

3. Patients with diabetes, patients receiving corticosteroids, patients on nonsteroidal antiinflammatory drugs, severe debilitating illness, patients less than 18 or more than 65 years of age.

4. Patients who were converted from laparoscopic to open cholecystectomy.

5. Patients in which common bile duct exploration or endoscopic retrograde cholangiopancreatography was done.

6. Patients operated in emergency hours.

7. Patients with psychiatric symptoms with persistence of abdominal pain, undergoing psychiatric management.

8. Antibiotic intake in the last 48 hours prior to surgery.

This was a postgraduate thesis work conducted in 2015, where data collection period was nearly 6 months. On scrutinising last 3 years data of our hospital records, we found that around 70 to 90 cases of laparoscopic cholecystectomy have been operated on an average in our hospital in General Surgery Department in previous 6 months duration. So it was decided to conduct the study among at least 70 cases. Author had collected data from surgery OPD twice weekly allotted to him during these 6 months data collection period from January 2015 to June 2015. During these 6 months period, we found 80 patients, who were eligible and also consented to participate in the study. These 80 patients were selected consecutively irrespective of age or sex for laparoscopic cholecystectomy and total 80 patients were randomised according to a computer generated block randomisation schedule equally into 40 patients in each two groups $(n=80 / 2=40)$. Group A (Antibiotic used) consisted of 40 patients and Group B (placebo, no antibiotic) also consisted of 40 patients. Group A patients $(n=40)$ received three doses of Cefazolin one just after the induction of anaesthesia and two after the operation. Group B ( $\mathrm{n}=40)$ patients received physiologic saline as placebo after the induction of anaesthesia. Patient demographics and clinical and surgical outcomes were recorded. Cefazolin (1 g) or normal saline was administered intravenously by the anaesthesiologist during induction of anaesthesia.

The method of data collection is based on direct observation of the study population in the postoperative period. All patients were followed up daily till discharge, then after 7 days (during stitch removal) and then 30 days following surgery to evaluate the surgical wound, and to look for signs and symptoms suggestive of any infection. Patients were studied at any time within 30 days following surgery if they presented with signs and symptoms suggestive of any infection.

Infective complications were defined as clinical features indicating the presence of infection with or without positive pathogen culture within 30 days of surgery. Infection sites were classified as-

1. Superficial wound infection (Signs of inflammation with or without discharges/collection).

2. Deep seated infection in the form of collection or abscesses in the abdominal cavity.

3. Distant infections away from the surgical site, such as respiratory and urinary tract infections.

In the event of any post-operative wound infection, microbiological investigations were performed by taking wound swab for aerobic and anaerobic culture and antibiotic sensitivity.

Statistical analyses were performed using chi-square test, Fisher's exact test (For cell frequency <5): KolmogorovSmirnov test and $t$ test for finding age distribution comparison. All analyses were conducted using Microsoft Excel 2010 and R statistical software, with a $\mathrm{P}$-value $<0.05$ considered as significant.

\section{RESULTS}

\section{Analysis}

All the patients were examined and investigated prior to their surgery, and the information needed was recorded.

Majority of patients were females $(n=52,65 \%)$. In Group A there were 22 females compared to 30 in Group B. No significant difference was noted among both groups based on gender distribution $(\mathrm{p}=0.12)$.

On performing Kolmogorov-Smirnov test for distribution similarity $(\mathrm{p}=0.68)$, results show similar age distribution in Group A and Group B. On performing t-test for equality of means, $p$ value was 0.38 , results show that mean age among Group A and B are similar. 
Total superficial wound infections were 6 in number (7.5\%). All 6 occurred between days 3-7 after the operation. Group A had 2(5\%) superficial wound infections at umbilical port site. Group B had 4(10\%) superficial wound infections at umbilical port site. The result was not statistically significant $(\chi 2=0.61, p$ value $=0.68)$. [Fig. No.1].

No significant difference was noted among both the groups $(p=0.68)$. In patients who developed post-op pus discharge from wound, necessary skin stitches were removed at the site of discharge. Patients were advised daily antibiotics and topical antibiotics. Infection subsided on followup.

Staph. aureus was the most common isolated organism in 2 out of 6 SSI cases, followed by coagulase-negative Staph. aureus in one case. Remaining SSI cases had no growth on pus culture sensitivity report. There were no cases of postoperative intra-abdominal collection or abscess, respiratory tract infections, urinary tract infections.

12 patients out of total 80 patients had intraoperative bile leakage. $7(17 \%)$ cases of biliary spillage among group B, $5(12 \%)$ in group A. Surgical site infection (SSI) occurred in 2 out of 12 patients with gallbladder rupture and 4 out of 68 patients without gallbladder rupture $(G B R)(p=0.22)$. Hence, there is no association between surgical site infection and intraoperative bile spillage.

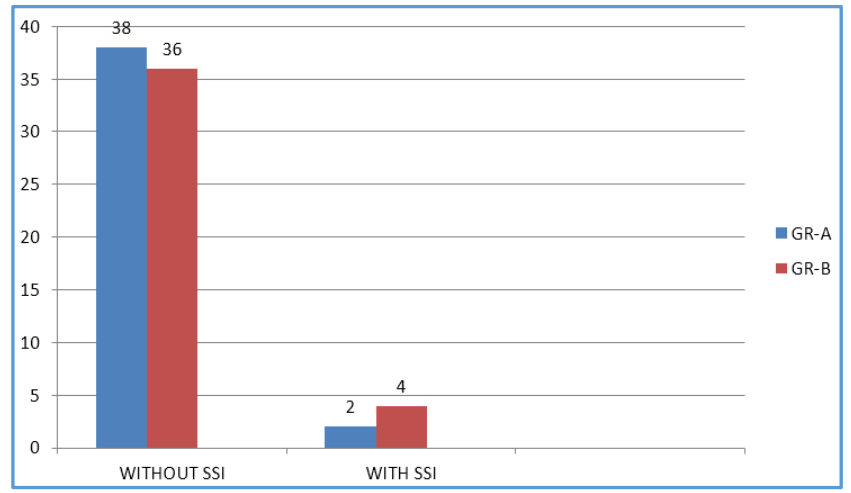

Figure 1. Distribution of Patients According to Wound Infection (Surgical Site Infection)

\section{DISCUSSION}

Though open cholecystectomy procedure is the most important part of surgical training, laparoscopic cholecystectomy is now the gold standard treatment for symptomatic cholelithiasis. Antibiotic prophylaxis is a standard component of biliary tract surgery, and there is about $50 \%$ decrease in the incidence of postoperative wound infection with the use of prophylactic antibiotics. ${ }^{19}$ However, these data cannot be extrapolated to include laparoscopic surgeries. As laparoscopic cholecystectomy is now the gold standard of management of patients with gallstones, evaluation of antibiotic prophylaxis and its indication for minimal invasive surgeries is warranted.

The average rate of SSIs for LC has been reported in the literature to be between $0.4 \%$ and $6.3 \%{ }^{7-9}$ which is lower than rates reported for open cholecystectomy.1,2

In the present study, the incidence of SSI in patients was $7.5 \%$ for the total study group, $5 \%$ for group A, and $10 \%$ for group $B$, and in agreement with previous studies, there was no significant difference in infection rate between the groups. All SSIs were superficial. Although antibiotic prophylaxis is considered standard protocol in open cholecystectomy as a means of reducing the incidence of infectious complications, its use is still debated in LC. Lippert and Gastinger ${ }^{14}$ performed a prospective population-based multicentre study to evaluate antimicrobial prophylaxis in laparoscopic and conventional cholecystectomy. They recommend that both laparoscopic and conventional open cholecystectomy be performed with adequate perioperative antimicrobial prophylaxis because patients receiving prophylaxis fared significantly better than those with no prophylaxis in terms of the rate of postoperative infections, other complications, reoperation, and mortality. Similarly, several other studies conclude that the use of prophylactic antibiotics in LC leads to a significant decrease in infectious complications. ${ }^{15-18}$ Conversely, many prospective studies have suggested that antibiotic prophylaxis is probably not required in elective LC, because the infection rate of LC is already low and the use of prophylactic antibiotics does not decrease the incidence of SSIs and other postoperative infection complications.

The positive bile culture rate in patients with gallbladder stones has ranged between $10 \%$ and $42.5 \%$ in previous studies.7,8,10,15-17 Perforation during gallbladder surgery is attributed to traction, grasping, dissection, and removal of the gallbladder and occurs in $11 \%$ to $35 \%$ of LCs.7,10,15,18 The effect of positive bacteria culture and bile or stone spilling due to perioperative gallbladder perforation on the occurrence of SSI infections is still controversial.

Shindholimath et $\mathrm{al}^{16}$ found that bactibilia was the most important predictor of wound infection in low-risk patients undergoing elective LC. They recommended prophylactic antibiotics to reduce the incidence of wound infection because it might not be possible to determine which patients have bactibilia by routine investigation. On the other hand, there was no relationship between the perioperative organisms isolated from the bile and the subsequent SSI in studies.7,10 Tocchi et al $^{10}$ in their study identified different microorganisms in pus culture in $10 / 11$ bile culture positive patients which had septic complications. However, Harling et $\mathrm{al}^{19}$ detected that all organisms isolated from the wound sites of patients who developed SSIs were skin commensals. They did not find a significant difference in rate of SSI between prophylactic antibiotic usage and mechanical isolation of the gallbladder with Endobag during extraction from the abdomen. Hamzaoglu et $\mathrm{al}^{20}$ also reported that the umbilical flora and the bile were not the source of the SSIs after laparoscopic surgery. It is not possible to assess the exact cause of SSI in the cases of cholecystectomy because there is no significant difference in the infection rate in the prophylactic and non-prophylactic groups. ${ }^{21}$

Low-risk patients were defined as those who did not have acute cholecystitis, a recent history of acute cholecystitis, common bile duct calculi, jaundice, immune suppression, or prosthetic implants. ${ }^{22}$ As patients converted to OC, with a history of previous biliary surgery, age $>60$ years, and bile spillage were reported to be at higher risk of post-operative infections, ${ }^{23-27}$ low risk patients should be also defined as those without any of these risk factors. On the contrary, highrisk patients were defined as those with the risk factors.

In a study conducted by Gaur and Pujahari, ${ }^{28}$ they concluded that the umbilicus is the commonest site for sepsis following laparoscopic cholecystectomy. This may be because the deep umbilical depression is sometimes difficult to clean. 
Also, it may be due to the routine protocol of our unit to extract the gallbladder through the umbilical port. Colizza and associates ${ }^{29}$ also stated that the umbilicus is the commonest site for sepsis in elective laparoscopic cholecystectomy.

Current guidelines do not recommend peri-operative antibiotics in patients undergoing cholecystectomy for lowor moderate-risk groups, owing to the modest risk of developing an SSI and costs to the healthcare system.(30) These guidelines are based, in part, on previous systematic reviews. ${ }^{31-34}$ In addition, there is a global campaign that aims to reduce inappropriate antibiotic administration to tackle the issue of emerging microbial resistance and other problems, such as increasing rates of Clostridium difficile infection. ${ }^{35,36}$ Despite this guidance and initiatives, between 20 and 80 percent of patients undergoing cholecystectomy with a low and moderate risk of SSI are still given antibiotics in nationally collected data sets. ${ }^{30,37,38}$ The adverse effects of antibiotic administration have been poorly studied. One RCT $^{39}$ reported on two patients who developed signs of an allergic reaction to the antibiotic given. It is surprising that, in the remaining studies, no anaphylactic reactions or $\mathrm{C}$. difficile infections were reported. It may be that these were not recorded specifically.

\section{CONCLUSION}

Laparoscopic cholecystectomy is now considered the gold standard in the management of symptomatic cholelithiasis. The role of prophylactic antibiotics in elective laparoscopic cholecystectomy is frequently debated. Hence, this study was intended to evaluate the efficacy of prophylactic antibiotics in low-risk patients undergoing elective laparoscopic cholecystectomy. There is no substitute for meticulous surgery and maintenance of strict asepsis while performing the operation. In the setting where the study was performed, aseptic precautions were taken in the best possible manner. Inadvertent lapse in asepsis might still have been possible due to the limited infrastructure available. In the Indian context where maximum hospitals have poor infrastructure, the result of this study might not always be applicable.

According to the results, there was no significant difference in wound infection rates in patient group given three doses of prophylactic antibiotic (Cefazolin), and in those receiving saline injection as placebo. Intraoperative bile spillage did not lead to increase in wound infection rates.

Hence, antibiotic prophylaxis is not necessary in low-risk patients with gallstone disease undergoing elective laparoscopic cholecystectomy to prevent postoperative infection-related complications.

\section{REFERENCES}

[1] Hoed DPT, Boelhouwer RU, Veen HF, et al. Infections and bacteriological data after laparoscopic and open gallbladder surgery. J Hosp Infect 1998;39(1):27-37.

[2] Chuang SC, Lee KT, Chang WT, et al. Risk factors for wound infection after cholecystectomy. J Formos Med Assoc 2004;103(8):607-12.

[3] Targarona EM, Balgue C, Knook MM, et al. Laparoscopic surgery and surgical infection. Br J Surg 2000;87(5):536-44.
[4] Mangram AJ, Horan TC, Pearson ML, et al. Guideline for prevention of surgical site infection, 1999. Hospital infection control practices advisory committee. Am J Infect Control 1999;20(4):205-78.

[5] Wittmann DH, Condon RE. Prophylaxis of postoperative infections. Infection 1991;19(Suppl 6):S337-44.

[6] Nichols RL. Preventing surgical site infections. Clin Med Res 2004;2(2):115-8.

[7] Chang WT, Lee KT, Chuang SC, et al. The impact of prophylactic antibiotics on postoperative infection complication in elective laparoscopic cholecystectomy: a prospective randomized study. Am J Surg 2006;191(6):721-5.

[8] Koc M, Zulfikaroğlu B, Kece C, et al. A prospective randomized study of prophylactic antibiotics in elective laparoscopic cholecystectomy. Surg Endosc 2003;17(11):1716-8.

[9] Mahatharadol V. A reevaluation of antibiotic prophylaxis in laparoscopic cholecystectomy: a randomized controlled trial. J Med Assoc Thai 2001;84(1):105-8.

[10] Tocchi A, Lepre L, Costa G, et al. The need for antibiotic prophylaxis in elective laparoscopic cholecystectomy: a prospective randomized study. Arch Surg 2000;135(1):67-70.

[11] Higgins A, London J, Charland S, et al. Prophylactic antibiotics for elective laparoscopic cholecystectomy: are they necessary? Arch Surg 1999;134(6):611-4.

[12] Page CP, Bohnen JM, Fletcher JR, et al. Antimicrobial prophylaxis for surgical wounds. Guidelines for clinical care. Arch Surg 1993;128(1):79-88.

[13] Kaiser AB. Overview of cephalosporin prophylaxis. Am J Surg 1988;155(5A):52-5.

[14] Lippert H, Gastinger J. Antimicrobial prophylaxis in laparoscopic and conventional cholecystectomy. Conclusions of a large prospective multicenter quality assurance study in Germany. Chemotherapy 1998;44(5):355-63.

[15] Uchiyama K, Kawai M, Onishi H, et al. Preoperative antimicrobial administration for prevention of postoperative infection in patients with laparoscopic cholecystectomy. Dig Dis Sci 2003;48(10):1955-9.

[16] Shindholimath VV, Seenu V, Parshad R, et al. Factors influencing wound infection following laparoscopic cholecystectomy. Trop Gastroenterol 2003;24(2):902.

[17] Dervisoglou A, Tsiodras S, Kanellakopoulou K, et al. The value of chemoprophylaxis against enterococcus species in elective cholecystectomy: a randomized study of cefuroxime vs ampicillin-sulbactam. Arch Surg 2006;141(12):1162-7.

[18] Sarli L, Pietra N, Costi R, et al. Gallbladder perforation during laparoscopic cholecystectomy. World J Surg 1999;23(11):1186-90.

[19] Ulualp K, Condon RE. Antibiotic prophylaxis for scheduled operative procedures. Infect Dis Clin North Am 1992;6(3):613-25. 
[20] Hamzaoglu I, Baca B, Boler DE, et al. Is umbilical flora responsible for wound infection after laparoscopic surgery? Surg Laparosc Endosc Percutan Tech 2004;14(5):263-7.

[21] Hassan AM, Nasr MM, Hamdy HE, et al. Role of prophylactic antibiotic in elective laparoscopic cholecystectomy. J Egypt Soc Parasitol 2012;42(1):129-34.

[22] Al-Ghnaniem R, Benjamin IS, Patel AG. Meta-analysis suggests antibiotic prophylaxis is not warranted in low-risk patients undergoing laparoscopic cholecystectomy. Br J Surg 2003;90(3):365-6.

[23] Dellinger EP, Gross PA, Barrett TL, et al. Quality standard for antimicrobial prophylaxis in surgical procedures. Infectious diseases society of America. Clin Infect Dis 1994;18(3):422-7.

[24] Diez J, Arozamena CJ, Ferraina P, et al. Relation between postoperative infections and gallbladder bile leakage during laparoscopic cholecystectomies. Surg Endosc 1996;10(5):529-32.

[25] Karayiannakis AJ, Polychronidis A, Perente S, et al. Laparoscopic cholecystectomy in patients with previous upper or lower abdominal surgery. Surg Endosc 2004;18(1):97-101.

[26] Annamaneni RK, Moraitis D, Cayten CG. Laparoscopic cholecystectomy in the elderly. JSLS 2005;9(4): 408-10.

[27] Simopoulos C, Botaitis S, Karayiannakis AJ, et al. The contribution of acute cholecystitis, obesity, and previous abdominal surgery on the outcome of laparoscopic cholecystectomy. Am Surg 2007;73(4):371-6.

[28] Shea JA, Healey MJ, Berlin JA, et al. Mortality and complications associated with laparoscopic cholecystectomy. A meta-analysis. Ann Surg 1996;224(5):609-20.

[29] Gaur A, Pujahari AK. Role of prophylactic antibiotics in laparoscopic cholecystectomy. MJAFI 2010;66(3):22830.
[30] Mahmoud SA, Khafagy WW, Omar W, et al. Antibiotic prophylaxis in elective laparoscopic cholecystectomy: a prospective study. Egyptian Journal of Surgery 2005;24(3).

[31] Graham HE, Vasireddy A, Nehra D. A national audit of antibiotic prophylaxis in elective laparoscopic cholecystectomy. Ann R Coll Surg Engl 2014;96(5):377-80.

[32] Choudhary A, Bechtold ML, Puli SR, et al. Role of prophylactic antibiotics in laparoscopic cholecystectomy: a meta-analysis. J Gastrointest Surg 2008;12(11):1847-53.

[33] Sanabria A, Dominguez LC, Valdivieso E, et al. Antibiotic prophylaxis for patients undergoing elective laparoscopic cholecystectomy. Cochrane Database Syst Rev 2010;12:CD005265.

[34] Zhou H, Zhang J, Wang Q, et al. Meta-analysis: antibiotic prophylaxis in elective laparoscopic cholecystectomy. Aliment Pharmacol Ther 2009;29(10):1086-95.

[35] Davey P, Brown E, Charani E, et al. Interventions to improve antibiotic prescribing practices for hospital inpatients. Cochrane Database Syst Rev 2013;4:CD003543.

[36] Blaser MJ. The microbiome revolution. J Clin Invest 2014;124(10):4162-5.

[37] Jaafar G, Persson G, Svennblad B, et al. Outcomes of antibiotic prophylaxis in acute cholecystectomy in a population-based gallstone surgery registry. Br J Surg 2014;101(2):69-73.

[38] Lundström P, Sandblom G, Osterberg J, et al. Effectiveness of prophylactic antibiotics in a population-based cohort of patients undergoing planned cholecystectomy. J Gastrointest Surg 2010;14(2):329-34.

[39] Matsui Y, Satoi S, Kaibori M, et al. Antibiotic prophylaxis in laparoscopic cholecystectomy: a randomized controlled trial. PLoS One 2014;9(9):e106702. 